\title{
CONSECUENCIAS DE LA OBESIDAD EN EL NIÑO Y EL ADOLESCENTE: UN PROBLEMA QUE REQUIERE ATENCIÓN
}

\author{
CONSEQUENCES OF OBESITY IN CHILDREN AND TEENAGERS: A PROBLEM THAT \\ REQUIRES ATTENTION
}

\author{
Reyna Liria ${ }^{1, a}$ \\ Instituto de Investigación Nutricional. Lima, Perú \\ a Nutricionista \\ Recibido: 26-06-12; Aprobado: 25-07-12
}

\begin{abstract}
RESUMEN
La obesidad es una pandemia mundial y los niños son un grupo vulnerable. En América se estimó que en el año 2010 el 15,2\% de los menores 18 años la podría sufrir. La obesidad en la niñez y la adolescencia tiene un impacto negativo en la salud y carga de enfermedades en esa etapa de vida y, posteriormente, en la adultez, cobrando un impacto negativo en la economía de un país debido a que se incrementa el riesgo de enfermedades crónicas, los gastos de salud y los costos indirectos como consecuencia de la enfermedad. El Perú está en un proceso de transición epidemiológica, donde no se ha solucionado los problemas de desnutrición y ya cuenta con altas tasas de obesidad infantil ( $10 \%$ en niños menores de cinco años); estando dentro de uno de los países con mayor incremento de la obesidad infantil en los últimos años a nivel Latinoamericano. La niñez y adolescencia son consideradas periodos críticos porque en estas etapas se instalan los hábitos alimentarios y de actividad física, y porque gran parte de los niños y adolescentes obesos seguirán siéndolo al llegar a adultos. Por ello, es crucial buscar estrategias e intervenciones que prevengan el sobrepeso y la obesidad infantil y del adolescente para así mejorar las condiciones de salud de un país.
\end{abstract}

Palabras clave: Sobrepeso; Obesidad; Niño; Adolescente; Causa; Impactos en la salud (fuente: DeCS BIREME).

\begin{abstract}
Obesity is a worldwide pandemic and children are a vulnerable group. In America, it was estimated that in 2010, $15.2 \%$ of 18 year-old children could suffer from this. Obesity in children and teenagers has a negative impact on health and on the load of diseases at this stage of life, and later on in adulthood, having a negative impact on the economy of a country due to the rise of risks of chronic diseases, health expenses and indirect costs as a result of the disease. Peru is going through an epidemiological transition, with unsolved malnutrition problems and high child obesity rates ( $10 \%$ of children under five), thus being one of the countries with a higher increase of child obesity in recent years in Latin America. Childhood and adolescence are considered critical periods because eating habits and physical activity start at this point; and because most obese children and teenagers will maintain those habits until they reach adulthood. For this reason, it is essential to seek strategies and interventions that prevent overweight and obesity among children and teenagers in order to improve the health conditions of a country.
\end{abstract}

Key words: Overweight; Obesity; Child; Adolescent; Causality; Impacts on health (source: MeSH NLM).

\section{INTRODUCCIÓN}

La obesidad, enfermedad que se puede iniciar desde la infancia, es considerada un problema de salud pública tanto en países desarrollados como en aquellos en vías de desarrollo. Desde 1997 un comité de expertos de la Organización Mundial de la Salud (OMS) hizo notar sobre la epidemia y advirtió que si no se hacía algo al respecto, millones de personas a nivel mundial estarían en riesgo de sufrir enfermedades crónicas (diabetes, hipertensión, ateroesclerosis, enfermedad cardiovascular, etc.) y que esto traería un alto impacto en la morbilidad y mortalidad ${ }^{(1,2)}$.

La OMS estimó en el 2005 que 1600 millones de adultos (mayores de 15 años) sufrían de sobrepeso, y 400 
millones de obesidad, cifra que en el 2012 alcanzaría a 2300 millones con sobrepeso y 700 millones con obesidad ${ }^{(3)}$. Este problema es particularmente grave en la infancia, la IOTF (International Obesity Task Force) estimó que 155 millones de niños (1 a 10 años, 2004) sufrían sobrepeso u obesidad (4) y la OMS estimó 43 millones (menores de 5 años, 2010) ${ }^{(5)}$. En América el 9,6\% de los niños en edad escolar tenían obesidad en el 2000 y se estimó que aumentaría a 15,2\% para el $2010^{(6)}$.

En Perú, el 10\% de niños menores de cinco años sufren de obesidad (más de dos desviaciones estándar peso/talla tomando la referencia de la OMS, Encuesta Nacional Demográfica y de Salud Familiar-ENDES 2007-2008) (7); mientras que $35 \%$ de las mujeres en edad fértil sufren de sobrepeso y $16 \%$ de obesidad (IMC entre 25 y 29,9 y mayor a 30,0 respectivamente; ENDES 2010) ${ }^{\left({ }^{8}\right)}$. La ENINBSC (Encuesta Nacional de Indicadores Nutricionales, Bioquímicos, Socioculturales Relacionados con las Enfermedades Crónicas Degenerativas, 2004-2005) mostró que el 31\% de hombres y $39 \%$ de mujeres mayores de 20 años tienen sobrepeso; mientras que $12 \%$ de hombres y $20 \%$ de mujeres tienen obesidad ${ }^{(9)}$. Un estudio que comparó la prevalencia de sobrepeso y obesidad en niños menores de 5 años en Latinoamérica, encontró que Perú tiene la mayor proporción de niños con sobrepeso (entre 1 a 2 desviaciones estándar de peso/ talla); además, ocupa el sexto lugar en obesidad (mayor o igual a 2 desviaciones estándar de peso/ edad) y es uno de los tres primeros países con mayor incremento de la obesidad en los últimos años en la región ${ }^{(10)}$.

Perú está viviendo cambios económicos importantes: en los últimos años ha mejorado la economía, ha disminuido la desigualdad y ha mejorado el ingreso per cápita. La economía está ubicada entre las de mejor desempeño en América Latina desde el 2002. Entre los años 2006 y 2008 el PBI (Producto Bruto Interno) creció 7,7; 8,9; y $9,8 \%$ respectivamente. Incluso, en el año 2009, mientras que las demás economías latinoamericanas mostraron una tasa negativa en promedio $(-2,3 \%)$, Perú mostró un $0,9 \%$ de incremento del $\mathrm{PBI}{ }^{(11)}$. El ingreso nacional bruto per cápita en 2009 se estimó en USD $7950{ }^{(12)}$. El último informe del PNUD (Programa de las Naciones Unidas para el Desarrollo, 2011), ubicó a Perú en uno de los cinco países de la región cuyos niveles de desigualdad interna disminuyeron en los últimos 10 años (usando el Índice de Desarrollo Humano ajustado por desigualdad) ${ }^{(13)}$. Este es un buen indicador para el país; sin embargo, este cambio positivo podría venir acompañado de un incremento del sobrepeso y obesidad en la población, incluyendo niños y adolescentes. En los últimos años, la obesidad en Perú es un problema más prevalente en población con mayores recursos económicos, no obstante, no deja de estar presente entre los pobres, en los que se ha observado un incremento de la obesidad entre los años 1991 y 2000 . En los no pobres, entre estos años, hubo un incremento de $32,4 \%$ en los niños menores de 5 años (de 10,8 a 14,3\%) y en los pobres extremos el incremento fue de $29,1 \%$ (de 7,9 a $10,2 \%$ ) ${ }^{(14)}$.

\section{NIÑEZ COMO PERIODO CRÍTICO PARA EL DESARROLLO DE OBESIDAD Y ENFERMEDADES CRÓNICAS}

Las prácticas de alimentación infantil influyen en el peso del niño. La lactancia ${ }^{(5,15-18)}$, en especial la exclusiva ${ }^{(19)}$, se ha asociado con menor riesgo de exceso de peso. Introducir alimentos diferentes a la leche materna antes de los cuatro meses de edad también se ha asociado con mayor obesidad infantil (5). La alimentación en la primera infancia es importante porque las preferencias alimentarias pueden ser innatas o aprendidas, por exposición ambiental repetida a alimentos no saludables: altos en grasa, azúcares y densamente energéticos ${ }^{(20)}$. Por otro lado, las golosinas y las entrecomidas altas en energía, y el incremento de las porciones han contribuido al incremento de la obesidad. De igual manera, las bebidas azucaradas (gaseosas, jugos, refrescos) que, aunque no tienen alta densidad energética, provee calorías que son asimiladas fácilmente por el organismo (21-22).

La actividad física es un componente importante en la regulación del gasto energético ${ }^{(18)}$. El desarrollo tecnológico (horas viendo televisión, computadora, video-juegos) ${ }^{(5,16,23-25)}$, y el ambiente escolar, se han asociado con menor gasto energético ${ }^{(26)}$. Las horas viendo televisión no solo podrían disminuir el tiempo de actividad física en el niño ${ }^{(24)}$, sino que la alta exposición a comerciales televisivos induciría al consumo de alimentos altamente energéticos ${ }^{(25,27)}$.

\section{CONSECUENCIAS A CORTO PLAZO DE LA OBESIDAD EN NIÑOS}

En los últimos años, la obesidad infantil se ha asociado con la aparición de alteraciones metabólicas (dislipidemias, alteración del metabolismo de la glucosa, diabetes, hipertensión, entre otras) a edades más tempranas (28). La Asociación Americana de Diabetes informó que el $85 \%$ de los niños con diagnóstico de diabetes tipo 2 tienen sobrepeso u obesidad; además, $10 \%$ de niños con obesidad podrían sufrir de tolerancia alterada a la 
glucosa. En adolescentes con obesidad, se encontró que el $25 \%$ presentan hipertensión, $39 \%$ bajos niveles de HDL, y $46 \%$ altos niveles de triglicéridos (frente a 4,18 y $17 \%$ en adolescentes con peso adecuado respectivamente). Del mismo modo, se ha descrito que adolescentes con oligomenorrea y obesidad están en mayor riesgo de desarrollar síndrome poliquístico ovárico. El $77 \%$ de niños obesos en China mostraron hígado graso y en EE.UU. se encontró que casi todos o todos los niños con esteatosis tenían obesidad (en un grupo reducido de niños). El apnea del sueño ha sido observada hasta en $50 \%$ de niños con obesidad en EE.UU. También se encontró dos veces mayor prevalencia de asma en niños con obesidad en Alemania, Israel y EE.UU. Otro problema del exceso de peso es el estrés mecánico, haciendo susceptible al niño de anormalidades ortopédicas y fracturas ${ }^{(4)}$.

Un niño obeso puede sufrir discriminación social, baja autoestima y depresión ${ }^{(4)}$. Se ha asociado la obesidad en la infancia y adolescencia con mayor probabilidad de presentar desórdenes de la alimentación (bulimia). Por otro lado, algunos estudios transversales han asociado a la obesidad en este periodo con burlas, bullying, marginación y pobres resultados académicos. Asimismo, se ha descrito que niños y adolescentes con obesidad informan mayores limitaciones funcionales, físicas y psicosociales lo que repercutiría en una baja calidad de vida debido al estigma social y las consecuencias de la obesidad sobre la salud ${ }^{(4,29)}$.

\section{CONSECUENCIAS A LARGO PLAZO DE OBESIDAD EN NIÑOS}

Se estima que $40 \%$, o más, de los niños, y $70 \%$ o más de los adolescentes con obesidad, llegarán a ser adultos obesos, con un impacto negativo en la salud y en la carga de enfermedad ${ }^{(30)}$. Niños y adolescentes con sobrepeso y obesidad tienen mayor riesgo de presentar enfermedades crónicas (enfermedad cardiovascular, ciertos tipos de cáncer, diabetes y asma) en la edad adulta, aunado a elevada mortalidad por estos problemas de salud ${ }^{(31)}$.

Las enfermedades agudas y crónicas asociadas con el exceso de peso no solo afectan la calidad de vida del individuo sino que también incrementan el costo individual, de la sociedad, costos de salud y baja productividad. Se ha estimado que la obesidad representa entre el 2 a $8 \%$ de los gastos en cuidados de salud (aunque se menciona que los datos son muy conservadores y podrían ser mayores). Asimismo, en $E E$. UU. se ha estimado que el gasto de un paciente obeso frente a un paciente con peso normal es $46 \%$ mayor en hospitalización, $27 \%$ mayor en consulta médica y $80 \%$ mayor en medicinas. Por otro lado, la obesidad igualmente trae como consecuencia costos indirectos por disminución de años perdidos por discapacidad, incrementada mortalidad antes de la jubilación, jubilación adelantada, pensiones por discapacidad y reducida productividad por ausentismo en el trabajo. Por todas las consecuencias indirectas de la obesidad, se piensa que estos costos son incluso mayores que los costos médicos ${ }^{(32)}$.

\section{CARGA DE ENFERMEDAD}

La carga de enfermedad es una medida de la brecha entre el estado de salud actual y una situación ideal en donde todos viven hasta una edad avanzada libre de enfermedad y discapacidad. El sobrepeso y la obesidad causan la muerte de alrededor de 2,8 millones de personas en el mundo en forma directa o indirecta, asociadas con enfermedades crónicas no transmisibles (2004) ${ }^{(1)}$. Se estima que $44 \%$ de la carga de diabetes, $23 \%$ de enfermedad coronaria isquémica y entre 7 a $41 \%$ de ciertos tipos de cáncer son atribuidos a la obesidad (21). Como consecuencia de la elevada carga de la enfermedad se ha mostrado que la obesidad aumenta la carga económica en un país ${ }^{(6,31)}$.

\section{CONCLUSIONES}

La obesidad se ha convertido en los últimos años en una pandemia mundial que afecta a la población de todos los grupos de edad. Este problema en la infancia y adolescencia se vuelve muy importante porque las prácticas de alimentación y actividad física en esta edad repercuten en el estado de salud del niño y, posteriormente, en la salud del adulto y en la economía del país. Perú es un país cuya economía ha mejorado en los últimos años, pero unido a esto también la prevalencia de sobrepeso y obesidad se ha incrementado. Es necesario buscar estrategias que ayuden a prevenir y disminuir el problema y evitar las consecuencias en la salud y economía para el individuo, familia, comunidad y país.

\section{Fuentes de financiamiento: autofinanciado.}

Conflictos de interés: el autor declara no tener ningún conflicto de interés. 


\section{REFERENCIAS BIBLIOGRÁFICAS}

1. World Health Organization (WHO). Obesity: preventing and managing the global epidemic. Report of a WHO consultation. Geneva: World Health Organization; 2000.

2. James WP. The epidemiology of obesity: the size of the problem. J Intern Med. 2008;263(4):336-52.

3. Organización Mundial de la Salud (OMS). Sobrepeso y obesidad. Nota descriptiva 311. Marzo 2011 [Internet]. Geneva: OMS; c2012 [citado el 12 de noviembre de 2011]. Disponible en: http://www. who.int/mediacentre/factsheets/fs311/ es/print.html

4. Lobstein T, Baur L, Uauy R. Obesity in children and young people: a crisis in public health. Obes Rev. 2004;5 Suppl 1:4104.

5. World Health Organization (WHO). Population-based prevention strategies for childhood obesity: report of a WHO forum and technical meeting. Geneva: WHO; 2010.

6. Wang Y, Lobstein T. Worldwide trends in childhood overweight and obesity. Int J Pediatr Obes. 2006;1(1):11-25.

7. Instituto Nacional de Estadística e Informática (INEI), Agencia de los Estados Unidos para el Desarrollo Internacional (USAID), Programa de Encuestas de Demografía y Salud (MEASURE). Presentación ENDES Continua, Ciclos IV y V (2007-2008), resultados preliminares de nutrición. Lima: INEI; 2009.

8. Instituto Nacional de Estadística e Informática (INEI), Agencia de los Estados Unidos para el Desarrollo Internacional (USAID), Ministerio de Economía y Finanzas. Perú: Encuesta Demográfica y de Salud Familiar - ENDES continua, 2010: informe principal. Lima: INEI; 2011.

9. Instituto Nacional de Salud (INS). Encuesta nacional de indicadores nutricionales, bioquímicos, socioeconómicos y culturales relacionados con las enfermedades crónicas degenerativas. Lima: INS; 2006.

10. Amigo H. Obesidad en el niño en América Latina: situación, criterios de diagnóstico y desafíos. Cad Saúde Publica. 2003,19(Supl 1):S163-S70.

11. Grupo del Banco Mundial. Perú: el país en datos [Internet]. Washington, DC: Banco Mundial; c2011 [citado el 17 de enero 2011]. Disponible en: http://www.bancomundial.org/es/country/peru
12. Organización Mundial de la Salud(OMS). Perú: datos estadísticos [Internet]. Geneva: OMS; c2012 [citado el 6 de diciembre de 2011]. Disponible en: http://www.who. int/countries/per/es/

13. Programa de las Naciones Unidas para el Desarrollo (PENUD). Informe sobre Desarrollo Humano 2011. Sostenibilidad y equidad: un futuro mejor para todos. Nueva York: Communications Development Incorporated; 2011.

14. Mispireta ML, Rosas AM, Velásquez JE, Velásquez, AG, Lescano AG, Lanata C. Transición nutricional en el Perú, 1991 2005. Rev Peru Med Exp Salud Publica. 2007;24(2):129-35.

15. Anderson PM, Butcher KF, Levine PB. Maternal employment and overweight children. J Health Econ. 2003;22(3):477504.

16. Aranceta-Bartrina J, Serra-Majem L, FozSala M, Moreno-Esteban B. Prevalencia de obesidad en España. Med Clin (Barc). 2005;125(12):460-6.

17. Kleiser C, Schaffrath RA, Mensink GB, Prinz-Langenohl R, Kurth BM. Potential determinants of obesity among children and adolescents in Germany: results from the cross-sectional KiGGS Study. BMC Public Health. 2009;9:46.

18. Elder JP, Arredondo EM, Campbell N, Baquero B, Duerksen S, Ayala G, et al. Individual, family, and community environmental correlates of obesity in Latino elementary school children. J Sch Health. 2010;80(1):20-30; quiz 53-5.

19. Rzehak P, Sausenthaler S, Koletzko S, Bauer CP, Schaaf B, von Berg A, et al. Period-specific growth, overweight and modification by breastfeeding in the GINI and LISA birth cohorts up to age 6 years. Eur J Epidemiol. 2009;24(8):449-67.

20. Drewnowski A, Specter SE. Poverty and obesity: the role of energy density and energy costs. Am J Clin Nutr. 2004;79(1):6-16.

21. Burns C, Jones SJ, and Frongillo EA. Poverty, household food insecurity and obesity in children. In: Waters E, Swinburn BA, Seidell JC and Uauy R eds. Preventing Childhood Obesity: Evidence Policy and Practice. Oxford: Wiley-Blackwell; 2010. p. 129-37.

22. Drewnowski A, Darmon N. The economics of obesity: dietary energy density and energy cost. Am J Clin Nutr. 2005;82(1 Suppl):S265-S73.
23. Du S, Mroz TA, Zhai F, Popkin BM. Rapid income growth adversely affects diet quality in China--particularly for the poor! Soc Sci Med. 2004;59(7):1505-15.

24. Amigo H, Bustos P, Erazo M, Cumsille P, Silva C. Factores determinantes del exceso de peso en escolares: un estudio multinivel. Rev Med Chil. 2007;135(12):1510-8.

25. Reilly JJ, Armstrong J, Dorosty AR, Emmett PM, Ness A, Rogers I et al. Early life risk factors for obesity in childhood: cohort study. BMJ. 2005;330(7504):1357.

26. Kain J, Concha F, Salazar G, Leyton B, Rodriguez Mdel P, Ceballos X, et al. Prevención de obesidad en preescolares y escolares de escuelas municipales de una comuna de Santiago de Chile: proyecto piloto 2006. Arch Latinoam Nutr. 2009;59(2):139-46.

27. Matijasevich A, Victora CG, Golding J, Barros FC, Menezes AM, Araujo CL, et al. Socioeconomic position and overweight among adolescents: data from birth cohort studies in Brazil and the UK. BMC Public Health. 2009;9:105.

28. Lobstein T, Baur LA, Jackson-Leach R. The Childhood Obesity Epidemic. In: Waters E, Swinburn BA, Seidell JC and Uauy R eds. Preventing Childhood Obesity: Evidence Policy and Practice. Oxford: Wiley-Blackwell; 2010. p. 3-14.

29. Field AE. Predictors and consequences of childhood obesity. In: Hu FB. Obesity Epidemiology. Oxford: Oxford University Press; 2008. p. 416-36.

30. Reinehr T, Kiess W, de Sousa G, StoffelWagner B, Wunsch R. Intima media thickness in childhood obesity: relations to inflammatory marker, glucose metabolism, and blood pressure. Metabolism. 2006;55(1):113-8.

31. Wang YC, McPherson K, Marsh T, Gortmaker SL, Brown M. Health and economic burden of the projected obesity trends in the USA and the UK. Lancet. 2011;378(9793):815-25.

32. Colditz GA, Wong C. Economics cost of obesity. In: Hu FB. Obesity Epidemiology. Oxford: Oxford University Press; 2008. p 261-74.

Correspondencia: Reyna Liria

Dirección: Av. La Molina 1885, Lima 12, Perú

Teléfono: (0511) 3496023

Correoelectrónico:rliria@hotmail.com 